\title{
Chitosan Derivatives/Calcium Carbonate Composite Capsules Prepared by the Layer-by-Layer Deposition Method
}

\author{
Takashi Sasaki, Misato Shimizu, Yusong Wu, and Kensuke Sakurai \\ Department of Materials Science and Engineering, Faculty of Engineering, University of Fukui, Fukui 910-8507, Japan
}

Correspondence should be addressed to Takashi Sasaki, sasaki@matse.fukui-u.ac.jp

Received 31 July 2007; Accepted 5 February 2008

Recommended by Xiaogong Wang

Core/shell capsules composed of calcium carbonate whisker core (rod-like shape) and chitosan/chitosansulfate shell were prepared by the layer-by-layer deposition technique. Two chitosan samples of different molecular weights $\left(M_{w}=9.7 \times 10^{4}\right.$ and $1.09 \times$ $10^{6} \mathrm{~g} \cdot \mathrm{mol}^{-1}$ ) were used as original materials. Hollow capsules were also obtained by dissolution of the core in hydrochloric acid. Electron microscopy revealed that the surface of the shell is rather ragged associated with some agglomerates. The shell thickness $l$ obeys a linear relation with respect to the number of deposited layers $m$ as $l=m d+a(a>0)$. The values of $d$ (thickness per layer) were 4.0 and $1.0 \mathrm{~nm}$ for the higher and lower $M_{w}$ chitosan materials, respectively, both of which are greater than the thickness of the monolayer. The results suggest that the feature of the deposition does not obey an ideal homogeneous monolayer-by-monolayer deposition mechanism. Shell crosslinked capsules were also prepared via photodimerization reaction of cinnamoyl groups after a deposition of cinnamoyl chitosan to the calcium carbonate whisker core. The degree of crosslink was not enough to stabilize the shell structure, and hollow capsule was not obtained.

Copyright (c) 2008 Takashi Sasaki et al. This is an open access article distributed under the Creative Commons Attribution License, which permits unrestricted use, distribution, and reproduction in any medium, provided the original work is properly cited.

\section{INTRODUCTION}

Development of encapsulation technique to fabricate nanosized core/shell or hollow particles is one of the most important and intriguing issues in the field of nanotechnology, especially because of its potential application to drug delivery systems. As for the shell material, biocompatibility is also important for such biomedical applications. Various approaches to prepare nanocapsules have been developed, and these may be classified into two categories, that is, capsules are formed either (1) by taking advantage of the self-assembly of amphiphilic molecules including micelles and vesicles, or (2) by using a core particle as a template. The former includes shell formation by polymerization and/or crosslinking of monomers in self-assembled system [1-4]. In general, the approaches of (1) are rather tricky, requiring sophisticated techniques to control the size and shape of the resulting capsule. In the latter approaches, on the other hand, the size and shape are determined by those of the core particles. In addition, if the core material is soluble in a suitable solvent, hollow capsules can easily be obtained. For example, ultrathin polymer shells have been formed on the surface of calcium carbonate particles via emulsion polymerization to prepare rod-like core/shell and hollow capsules [5, 6].

The layer-by-layer deposition method, which belongs to the category (2), is one of the most powerful and versatile techniques to fabricate thin complex multilayers with controllable thicknesses and geometries $[7,8]$. By this technique, multilayered films can be prepared by alternative adsorption of oppositely charged polyelectrolytes on a substrate or particle surface. Various polyelectrolytes have been demonstrated to give multilayered films on the surface of various particles [8-15]. Hollow capsules can also be prepared by dissolution of the core materials in appropriate solvents $[9,10,14]$. Also, it is possible in some cases to substitute the original core for another material. For example, Shchukin et al. prepared ferrite and magnetite particles inside hollow capsules that had been prepared by the layer-by-layer method [16].

In this paper, we report preparations of biocompatible core/shell capsules by the layer-by-layer technique. The shells were formed with alternative deposition of chitosan (cationic polyelectrolyte) and chitosansulfate (anionic polyelectrolyte) on the surface of calcium carbonate whisker as a core. The core can be removed being dissolved in hydrochloric acid to 
obtain hollow capsules. As the whisker particles have a rodlike shape, the obtained capsules are rod-like. Such a rod-like shape is unique in the sense that the particle can be oriented by elongation or under flow. Chitosan is obtained by acetylation of an abundant natural polysaccharide, chitin. There has been a growing interest to chitosan as a biomedical material because of its biocompatibility and biodegradability with little toxicity. To stabilize the core/shell structure, we also tried to prepare shell crosslinked capsules: cinnamoyl chitosan was deposited onto the whisker core, and the crosslinking via photodimerization of the cinnamoyl groups was performed by UV irradiation.

\section{EXPERIMENTAL METHODS}

\subsection{Materials}

Chitosan $\left(M_{w}=9.7 \times 10^{4}\right.$ and $\left.1.09 \times 10^{6} \mathrm{~g} \cdot \mathrm{mol}^{-1}\right)$ was purified by dissolving it in $1.0 \%$ acetic acid aqueous solution, followed by precipitation in $10 \%$ aqueous solution of sodium hydroxide. The precipitate was washed with distilled water, methanol, acetone, and with N,N-dimethyl formamide (DMF). Calcium carbonate whisker (BS-P, Maruo Calcium Co., Japan) was supplied from the manufacturer, of which the diameter ranged from 300 to $600 \mathrm{~nm}$ and the length ranged from 1.0 to $3.0 \mu \mathrm{m}$. The surface of BS-P whisker was covered with a thin layer of calcium phosphate via phosphoric acid treatment.

\subsection{Preparation of chitosansulfate}

Sulfonic groups were introduced at either the hydroxyl groups or amino groups in 2-amino-2-deoxy-D-glucopyranose unit of chitosan in the following method. Into a flask containing $25 \mathrm{ml}$ of DMF was added dropwise $16.5 \mathrm{ml}$ of chlorosulfonic acid in an ice-water bath, and immediately added $2.5 \mathrm{~g}$ of the purified chitosan. The mixture was stirred at room temperature for 24 hours. After the reaction was completed, the mixture was poured into $400 \mathrm{~mL}$ of methanol with stirring. The precipitate was separated and washed with methanol. The obtained product was dissolved in distilled water, and the solution was neutralized with $20 \%$ aqueous solution of sodium hydroxide. Then, the solution was subject to dialysis with distilled water for 36 hours. Final product chitosansulfate was obtained by freeze-drying the dialyzed solution. IR (KBr) $810(\mathrm{C}-\mathrm{O}-\mathrm{S}), 1122\left(\mathrm{SO}_{2}\right) \mathrm{cm}^{-1}$. Elementary analysis revealed the number of sulfonic groups per repeating unit of chitosan to be 0.86 .

\subsection{Layer-by-layer deposition}

Layer-by-layer deposition of chitosan/chitosansulfate was executed according to the membrane filtration method by Voigt et al. [17], that is, the suspended state of particles were maintained throughout the deposition-filtration-lavagefiltration cycles to avoid any damages of capsules due to particle aggregation. Into $500 \mathrm{~mL}$ of an aqueous solution consisted of sodium chloride $\left(0.50 \mathrm{~mol} \mathrm{~L}^{-1}\right)$ and acetic acid $(1.0 \% \mathrm{v} / \mathrm{v})$ was dissolved $2.5 \mathrm{~g}$ of chitosan (referred to as $\mathrm{A})$.
An aqueous solution of chitosansulfate $\left(0.53 \mathrm{~g} \mathrm{~L}^{-1}\right)$ that contained $0.50 \mathrm{~mol} \mathrm{~L}^{-1}$ of sodium chloride was prepared (referred to as B). One gram of calcium carbonate whisker (BSP) was suspended in $20 \mathrm{~mL}$ of distilled water with gentle sonication, and $5 \mathrm{~mL}$ of the chitosan solution A was added. The suspension was stirred for 5 minutes for deposition of chitosan salt layer on the whisker surface. The suspended particles were washed by adding distilled water continuously over a filter with constant stirring so as to keep a well-suspended state even during the filtration. Subsequently, $5 \mathrm{~mL}$ of the solution $\mathrm{B}$ was added to the suspension, which was stirred for 5 minutes for deposition of chitosansulfate layer. The suspension was then washed with distilled water in the same manner as above. The cycle of deposition with solutions A and $B$ was repeated 2-7 times to prepare capsules with different total numbers of deposited layers $m(m=4-14)$.

\subsection{Preparation of hollow particles}

The core/shell capsules were converted to hollow particles by adding hydrochloric acid to their aqueous suspensions (10 wt $\%)$ to dissolve the core part (calcium carbonate). The acid suspensions were stirred for 2 hours, of which the $\mathrm{pH}$ value was kept below 1.0. The particles were separated by centrifugation and washed with water several times. The final products were dried under vacuum for more than 48 hours at room temperature.

\subsection{Preparation of cinnamoyl chitosan}

Acylation of chitosan with cinnamoyl chloride was performed according to the method described in [18]. The cinnamoyl group was introduced at either of the two hydroxyl groups in the repeating unit of chitosan. To $30 \mathrm{~mL}$ of methanesulfonic acid was added $2 \mathrm{~g}$ of the purified chitosan. The mixture was cooled in an ice-water bath, and was added $0.4 \mathrm{~g}$ of cinnamoyl chloride. Subsequently, the solution was stirred at room temperature for 2 hours, and then, it was poured into $500 \mathrm{~mL}$ of methanol. The precipitate was separated by filtration, washed with methanol and with acetone. The product was then reprecipitated in ammonium hydroxide aqueous solution ( $\mathrm{pH}=8.0)$. Finally, the separated product was washed with water, methanol, and acetone, followed by filtration and drying under vacuum at room temperature for 24 hours. IR (KBr) 1705 (ester carbonyl), 1636 (disubstituted alkene) $\mathrm{cm}^{-1}$. Quantitative analysis of UV absorption at $280 \mathrm{~nm}$ in DMF/water (1/1) containing acetic acid $(1.0 \% \mathrm{v} / \mathrm{v})$ solution revealed that the number of cinnamoyl groups was 0.15 per repeating unit of chitosan.

\subsection{Deposition of cinnamoyl chitosan on the whisker surface}

Into $500 \mathrm{~mL}$ of an aqueous solution consisted of sodium chloride $\left(0.50 \mathrm{~mol} \mathrm{~L}^{-1}\right)$ and acetic acid $(1.0 \% \mathrm{v} / \mathrm{v})$, was dissolved $2.5 \mathrm{~g}$ of cinnamoyl chitosan. The solution was added to an aqueous suspension of BS-P $\left(50 \mathrm{~g} \mathrm{~L}^{-1}\right)$, and it was stirred for 10 minutes for deposition of cinnamoyl chitosan single layer on the whisker surface. Subsequently, UV 
irradiation $(\lambda=254 \mathrm{~nm})$ to the suspension was executed for 48 hours at room temperature with constant stirring.

\subsection{Microscopy}

To investigate the morphology and sizes of the capsules, transmission electron microscopy (TEM) was done by using a Jeol JEM-2000FXII operated with an acceleration voltage of $200 \mathrm{kV}$. No shadowing was applied. Scanning electron microscopy (SEM) was also done by using a Hitachi S-2300.

\subsection{Evaluation of the core/shell ratio}

Compositions (core/shell ratio) of the capsules were evaluated by volumetric analysis. A finite mass of the capsule was immersed in hydrochloric acid with stirring until the core material was completely dissolved. Hydrochloric acid and water in the obtained solution were evaporated and residual precipitate was redissolved in deionized water. Calcium ion content in this solution was determined by chelatometric titration, and the ratio of the mass of the shell to that of the whole capsule $m_{s} / m_{t}$ was evaluated.

\section{RESULTS AND DISCUSSION}

TEM observations confirmed that thin shells of chitosan/ chitosansulfate are formed by the layer-by-layer technique. Figures 1, 2, and 3 show TEM images for the core/shell and hollow capsules prepared by the layer-by-layer deposition. For core/shell particles, thin outer shells are discernible around the dark core part. Also, formation of hollow capsules is clearly seen, confirming that the deposition of the outer layer occurred. It is also noticed that the surface of the layer seems to be rather rough and ragged, and that the layer contains some agglomerates. These features are more clearly seen for the hollow samples. Occasionally for some of the core/shell particles, the core part was lost resulting in partially or totally hollow particles as shown in Figures 1(d), 2(c) and $2(\mathrm{~d})$. This phenomenon was observed more frequently for samples with higher $m$. This may be due to slight solubility of the calcium carbonate in the acidic aqueous solvent (acetic acid aqueous solution) that was used in the deposition process.

Hollow particles were successfully obtained by the treatment with hydrochloric acid only for the higher molecular weight $\left(M_{w}\right)$ chitosan/chitosansulfate $\left(1.09 \times 10^{6} \mathrm{~g}\right.$. $\left.\mathrm{mol}^{-1}\right)$. The shell prepared from the lower $M_{w}$ chitosan/chitosansulfate $\left(9.7 \times 10^{4} \mathrm{~g} \cdot \mathrm{mol}^{-1}\right)$ was probably lost by dissolution into the hydrochloric acid aqueous solution during the core-removing process. However, the unintentional hollow capsules mentioned above (yielded without the hydrochloric acid treatment) were partially obtained even for the lower $M_{w}$ material.

Table 1 shows the layer thickness $l$ and diameter $D$ of the obtained core/shell and hollow capsules. The values presented in this table were obtained by averaging over a few hundreds of sampling points in TEM micrographs obtained with higher magnifications as typically shown in Figure 3. The standard deviations for $l$ are fairly large: this is due to the ragged feature of the shell. On the other hand, the large standard deviations for $D$ are due to the original size distribution of BS-P as we reported previously [5].

The layer thickness $l$ increases with increasing the total number of depositions (deposited layers) $m$. However, $l$ is not directly proportional to $m$, and an expression $l=m d+a(a>$ 0 ) describes the data well. This means that the deposited amount in the first deposition cycle is greater than that in the later cycles. The deposition mechanism onto the surface of BS-P may be different from that of the succeeding deposition, reflecting the different chemistry of the surface from that for the deposited chitosan/chitosansulfate surface. Linear regression analysis revealed average shell thicknesses per layer to be $d=4.0$ and $1.0 \mathrm{~nm}$ for the higher and lower molecular weights of the original chitosan, respectively. These values are much higher than the thickness of a chitosan monolayer (a few angstroms). These results suggest that the deposition does not occur by a homogeneous monolayer-by-monolayer mechanism and that the opposite charges in the deposited layer are not distributed uniformly, which may lead to some aggregation in the succeeding deposition process. Also, the estimated values of $d$ suggest that the aggregation during the deposition occurs to less extent for the lower $M_{w}$ material than for the higher one.

The large $d$ values seem to be consistent with the TEM observation showing some agglomerates in the deposited layer. Our previous circular dichroism study revealed that the chitosan chains are rather rigid in aqueous acidic solution, taking a helical conformation [18], which might prevent to form a homogeneous flat layer with uniformly distributed charges upon depositing. The helical conformation may have tendency to form an ordered structure, which also promotes the molecular aggregation [19].

The values of the core/shell composition $m_{s} / m_{t}$ in the capsules are qualitatively consistent with the shell thickness (Table 1). However, thickness calculated from the obtained $m_{s} / m_{t}$ values is much greater than the $d$ values evaluated from the TEM observation, for example, for the higher $M_{w}$ sample with $m=14$, the calculated thickness is $130 \mathrm{~nm}$. This is probably due to residual chitosan and chitosansulfate that was not deposited on the whisker particles. It seems to be rather difficult to remove such excess polymer impurities by the present preparation method.

From the above results, we conclude that the present deposition mechanism is far from an ideal process of a monolayer-by-monolayer accumulation caused exclusively by non-bonded interactions between the oppositely charged sites. Nevertheless, the layer-by-layer method was revealed to be available to fabricate capsules possessing chitosan/chitosansulfate shell.

Slightly larger apparent diameters $D$ were observed for the hollow samples than for the core/shell ones as shown in Table 1. This may be due to deformation of the shell on removing the core. If the hollow shell collapsed into a completely flat geometry, the observed width (apparent diameter) would be greater than the diameter of the original core/shell particle by a factor of $\pi / 2 \cong 1.57$. However, Table 1 shows much less increase in $D$ for the hollow particles, suggesting that they are not completely flat. This can be 


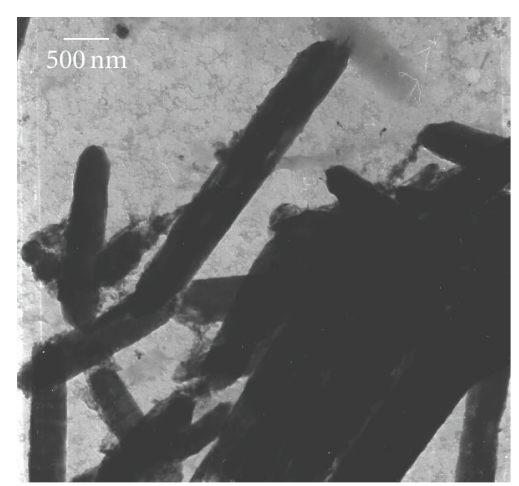

(a)

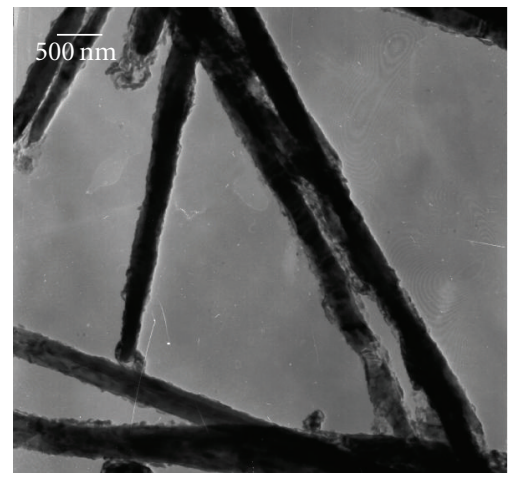

(c)

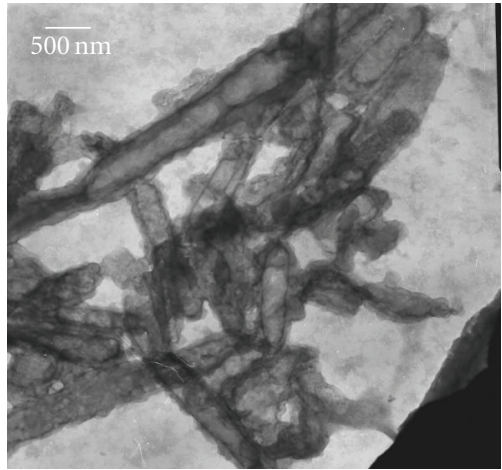

(b)

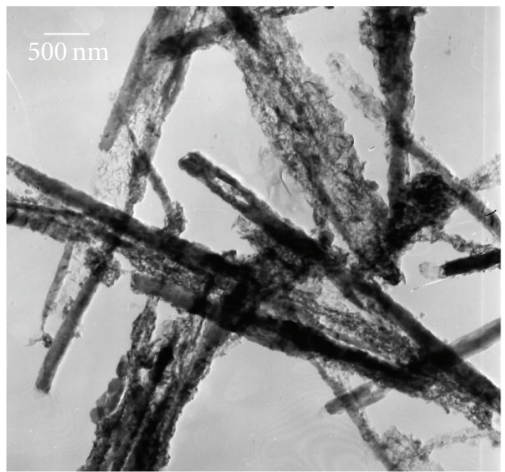

(d)

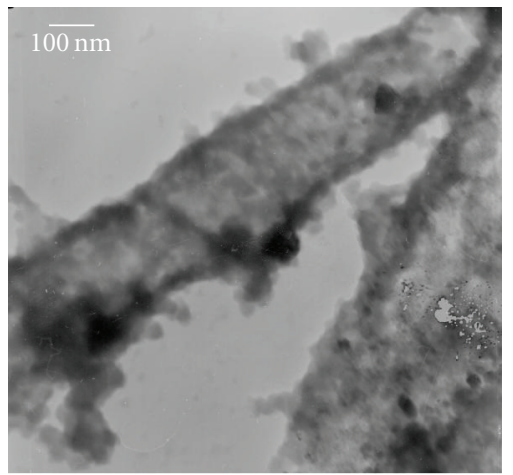

(e)

Figure 1: Transmission electron micrographs for capsules prepared from the higher $M_{w}$ chitosan/chitosansulfate by the layer-by-layer deposition method. (a) Core/shell capsules with $m=4$, (b) hollow capsules with $m=4$, (c)-(d) core/shell capsules with $m=14$, and (e) hollow capsules with $m=14$.

confirmed by SEM images presented in Figure 4. Such appearance of the hollow particles indicates that the shell has some rigidity to retain the original shape of core/shell capsule to some extent in contrast to the hollow shells composed of other synthetic polyelectrolytes such as poly(sodium 4styrenesulfonate) (PSS) and poly(allylamine hydrochloride) (PAH) system, of which the appearance looks like a deflated balloon with wrinkles $[10,11]$. The rigid feature of this chitosan/chitosansulfate shell may be originated from the inherent rigidity of the polymer chain, that is, a polysaccha- ride chitosan has rather high chain rigidity. In addition, once the chitosan and chitosansulfate chains are deposited on the surface, no swelling with the medium may occur, thus, the vitrification of shell immediately occurs after the deposition, resulting in rigidity of the shell.

Another cause for the less increase in $D$ on removing the core could be shrinkage of the whole capsule. Significant shrinkage has been reported to occur for the polymer shells deposited to the BS-P whisker surface via emulsion polymerization [5]. This is associated with a thickening of the shell. 


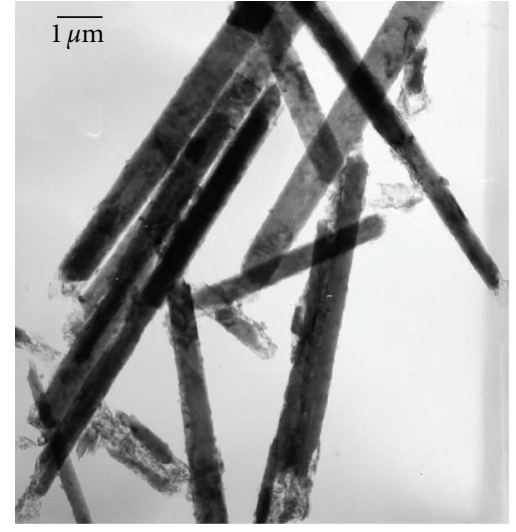

(a)

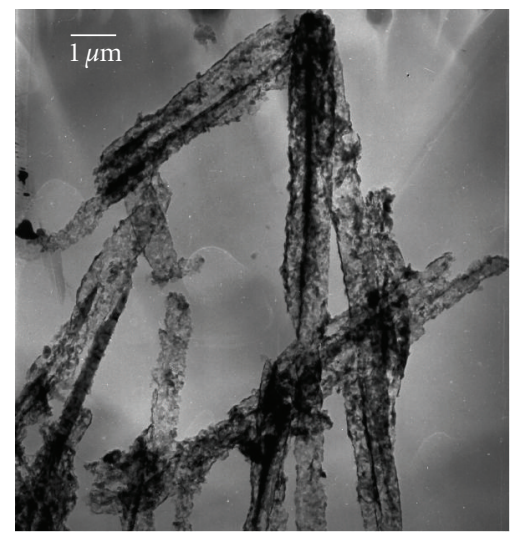

(c)

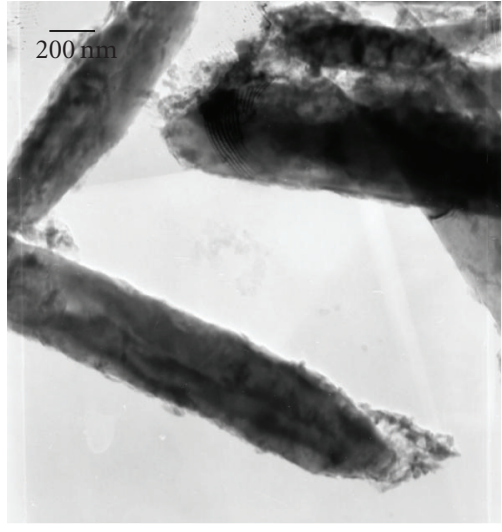

(b)

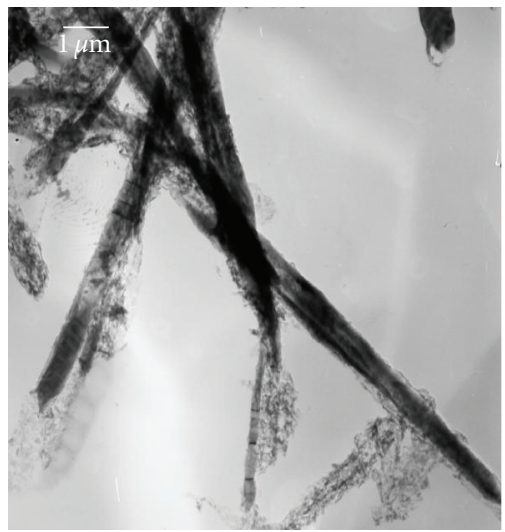

(d)

FIgURE 2: Transmission electron micrographs for capsules prepared from the lower $M_{w}$ chitosan/chitosansulfate by the layer-by-layer deposition method. (a) Core/shell capsules with $m=4$, (b) $m=8$, and (c)-(d) $m=12$.

For the present capsules with chitosan/chitosansulfate shells, however, shell thickening is not observed clearly in Table 1. It is likely that no significant shrinkage occurs for the present capsules. The absence of shrinkage may be partially due to the rigidity of the shell as mentioned above. There may be an essential difference in the deposition mechanism between the seed emulsion polymerization [5] and the present layerby-layer method: in the former, some configurational instability is frozen in during polymerization-vitrification process and relaxation (stress release) occurs when the core is removed, while in the latter process, stable configuration could be achieved to some extent in the solution state prior to deposition, and therefore, no structural relaxation occurs on removing the core.

Figure 5 shows transmission electron micrographs for the capsules with the UV irradiated cinnamoyl chitosan shells. The deposition was successful, and the mean layer thickness of the core/shell capsules was $33 \pm 18 \mathrm{~nm}$. This is even higher than the thickness $30 \mathrm{~nm}$ of the shell prepared by the layer-by-later method with $m=4$ (Table 1). It is not clear whether this is due to an error (standard deviation is $18 \mathrm{~nm}$ ) or to an intrinsic deposition feature characteristic to cinnamoyl chitosan. Unfortunately, no hollow capsules were obtained even for the higher $M_{w}$ material. This may be due to insufficient degree of crosslink of the shell, that is, the crosslinking photoreaction did not occur enough to stabilize the shell structure, and the cinnamoyl chitosan was probably dissolved away in the hydrochloric acid in the core-removing process.

In an acetic acid aqueous solution $(1.0 \% \mathrm{v} / \mathrm{v})$ of cinnamoyl chitosan, the photodimerization reaction was confirmed by a significant decrease in UV absorption at $280 \mathrm{~nm}$ (characteristic to cinnamoyl group) and by an IR absorption band at $1458 \mathrm{~cm}^{-1}$ (cyclobutane ring) after UV irradiation $(\lambda=254 \mathrm{~nm})$ for 24 hours at room temperature. However, for the deposited shell, only a slight signal was observed at $1458 \mathrm{~cm}^{-1}$, indicating that the reaction could occur to less extent than in the solution state. Thus, it may be difficult to yield a high degree of crosslink by the present photoreaction in the solid shell. Once the cinnamoyl chitosan is deposited on the whisker, its conformational motion is frozen, and photodimerization reaction occurs only for the pairs of cinnamoyl groups which accidentally take conformations suitable for the reaction. In addition, the reaction contains intrachain reactions, which results in no crosslink. 


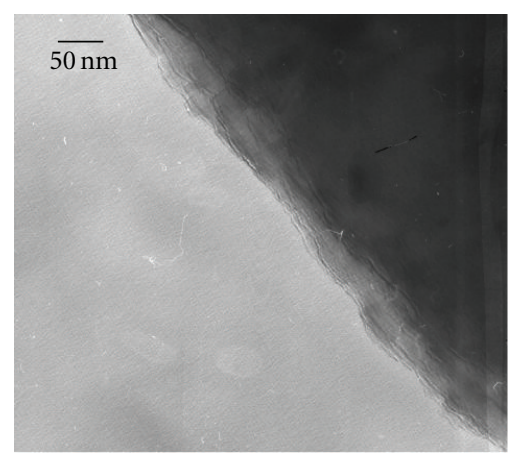

(a)

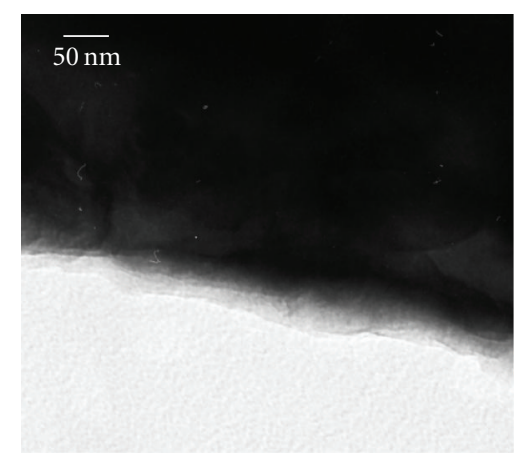

(c)

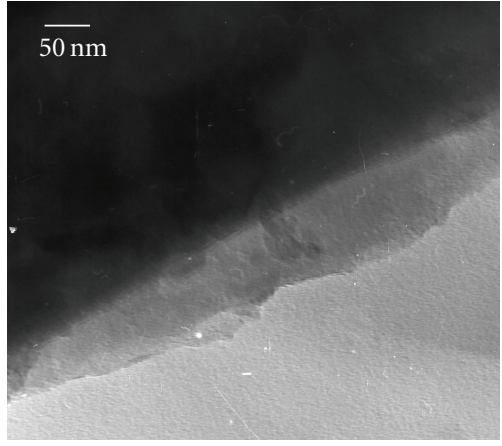

(b)

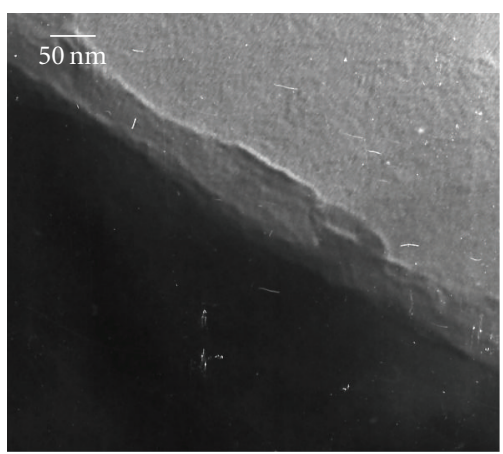

(d)

Figure 3: Transmission electron micrographs for core/shell capsules of (a) $m=4$ with the higher $M_{w}$, (b) $m=14$ with the higher $M_{w}$, (c) $m=4$ with the lower $M_{w}$, and (d) $m=12$ with the lower $M_{w}$.

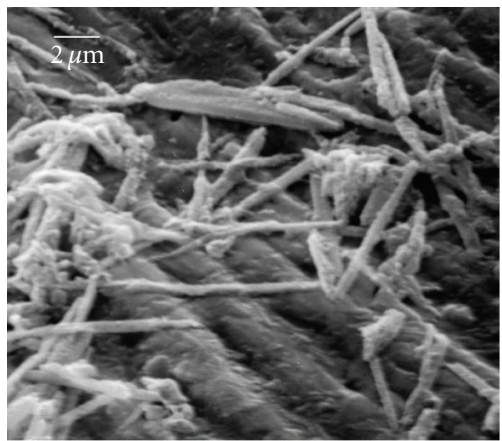

(a)

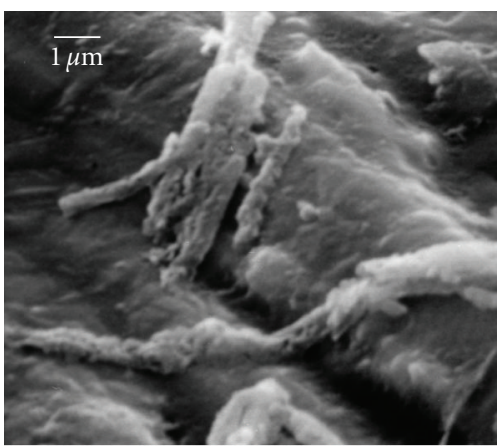

(b)

FIGURE 4: Scanning electron micrographs for hollow capsules prepared from the lower $M_{w}$ chitosan/chitosansulfate by the layer-by-layer deposition method $(m=12)$. These hollow particles were unintentionally obtained without the treatment with hydrochloric acid (probably the core was accidentally dissolved away during the deposition processes).

\section{CONCLUSIONS}

We have successfully prepared core/shell nanocapsules possessing biocompatible outer shells of chitosan/chitosansulfate by the layer-by-layer deposition method. Hollow capsules were obtained only for the higher $M_{w}$ chitosan/chitosansulfate shells. Electron microscopy revealed that the thickness of the shell $l$ increases almost linearly with increasing $m$ though it is not directly proportional to $m$, and that the thickness per layer is greater than that of the chitosan monolayer. These results as well as the ragged surface morphology of the deposited layers with agglomerates suggest that the deposition mechanism is not exactly an ideal monolayer-by-monolayer process. The rigidity of chitosan chain may be responsible for the above feature of deposition. Nevertheless, our results have demonstrated a great possibility to fabricate nanocapsules possessing outer shells of rigid polysaccharides such as chitosan/chitosan derivatives by the 
TABLE 1: Shell thickness $l$, diameter $D$, and core/shell composition of the capsules prepared by the layer-by-layer deposition method.

\begin{tabular}{|c|c|c|c|c|c|c|c|}
\hline & $M_{w}{ }^{(1)}$ & $m$ & $l(\mathrm{~nm})$ & $\sigma_{1}{ }^{(2)}(\mathrm{nm})$ & $D(\mathrm{~nm})$ & $\sigma_{D}^{(3)}(\mathrm{nm})$ & $m_{s} / m_{t}{ }^{(4)}$ \\
\hline Core/shell & $\mathrm{H}$ & 4 & 30 & 12 & 397 & 54 & 0.09 \\
\hline Hollow & $\mathrm{H}$ & 4 & 42 & 4 & 409 & 132 & - \\
\hline Core/shell & $\mathrm{H}$ & 8 & 48 & 9 & 320 & 55 & 0.11 \\
\hline Hollow & $\mathrm{H}$ & 8 & 34 & 8 & 328 & 55 & - \\
\hline Core/shell & $\mathrm{H}$ & 10 & 63 & 14 & 323 & 57 & - \\
\hline Hollow & $\mathrm{H}$ & 10 & 40 & 13 & 345 & 48 & - \\
\hline Core/shell & $\mathrm{H}$ & 14 & 69 & 12 & 380 & 39 & 0.16 \\
\hline Hollow & $\mathrm{H}$ & 14 & 51 & 18 & 408 & 64 & - \\
\hline Core/shell & $\mathrm{L}$ & 4 & 14 & 4 & - & - & 0.08 \\
\hline Core/shell & $\mathrm{L}$ & 8 & 18 & 6 & - & - & 0.07 \\
\hline Core/shell & $\mathrm{L}$ & 12 & 22 & 8 & - & - & 0.10 \\
\hline
\end{tabular}

${ }^{(1)} \mathrm{L}: M_{w}=9.7 \times 10^{4} \mathrm{~g} \cdot \mathrm{mol}^{-1}, \mathrm{H}: M_{w}=1.09 \times 10^{6} \mathrm{~g} \cdot \mathrm{mol}^{-1}$.

(2) Standard deviation for $l$.

${ }^{(3)}$ Standard deviation for $D$.

${ }^{(4)}$ Ratio of the mass of the shell to that of the whole capsule evaluated by chelatometric titration.

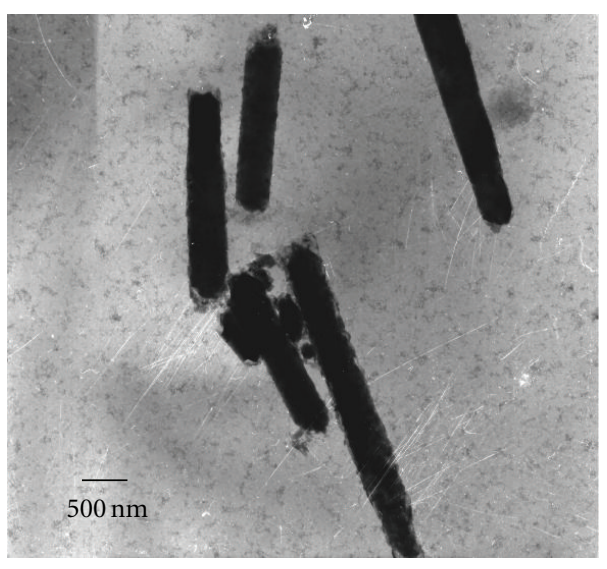

FIgURE 5: Transmission electron micrograph for shell crosslinked capsules prepared by UV irradiation after deposition of cinnamoyl chitosan. The core was not removed.

layer-by-layer deposition method. The photo-crosslinking of cinnamoyl chitosan shell by UV irradiation was not enough to stabilize the shell structure. An alternative method using interfacial reactions by crosslinking monomers such as diisocyanate compounds [4] may be preferable to achieve higher degree of crosslink.

\section{ACKNOWLEDGMENTS}

This work was supported by a Grant-in-Aid for Scientific Research (Grant no. 18550107) from the Ministry of Education, Culture, Sports, Science, and Technology of Japan. The authors thank Maruo Calcium Co, Japan, for supplying the calcium carbonate whisker BS-P. The authors also thank Hiroto Mizuuchi and Masashi Kawaminami for their help in conducting experiments.

\section{REFERENCES}

[1] M. Dvolaitzky, M. A. Guedeau-Boudeville, and L. Léger, "Polymerization-induced shrinkage in giant butadienic lipid vesicles," Langmuir, vol. 8, no. 11, pp. 2595-2597, 1992.

[2] K. B. Thurmond II, T. Kowalewski, and K. L. Wooley, "Shell cross-linked knedels: a synthetic study of the factors affecting the dimensions and properties of amphiphilic coreshell nanospheres," Journal of the American Chemical Society, vol. 119, no. 28, pp. 6656-6665, 1997.

[3] J. Hotz and W. Meier, "Vesicle-templated polymer hollow spheres," Langmuir, vol. 14, no. 5, pp. 1031-1036, 1998.

[4] D. Crespy, M. Stark, C. Hoffmann-Richter, U. Ziener, and K. Landfester, "Polymeric nanoreactors for hydrophilic reagents synthesized by interfacial polycondensation on miniemulsion droplets," Macromolecules, vol. 40, no. 9, pp. 3122-3135, 2007.

[5] T. Sasaki, T. Kitagawa, S. Sato, S. Irie, and K. Sakurai, "Core/shell and hollow polymeric capsules prepared from calcium carbonate whisker," Polymer Journal, vol. 37, no. 6, pp. 434-438, 2005.

[6] T. Sasaki, S. Kawagoe, H. Mitsuya, S. Irie, and K. Sakurai, "Glass transition of crosslinked polystyrene shells formed on the surface of calcium carbonate whisker," Journal of Polymer Science B, vol. 44, no. 17, pp. 2475-2485, 2006.

[7] G. Decher, "Layered nanoarchitectures via directed assembly of anionic and cationic molecules," in Comprehensive Supramolecular Chemistry, J.-P. Sauvage and M. W. Hosseini, Eds., vol. 9, pp. 507-528, Pergamon Press, Oxford, UK, 1996.

[8] M.-K. Park, C. Xia, R. C. Advincula, P. Schütz, and F. Caruso, "Cross-linked, luminescent spherical colloidal and hollowshell particles," Langmuir, vol. 17, no. 24, pp. 7670-7674, 2001.

[9] H. Duan, D. Chen, M. Jiang, et al., "Self-assembly of unlike homopolymers into hollow spheres in nonselective solvent," Journal of the American Chemical Society, vol. 123, no. 48, pp. 12097-12098, 2001.

[10] E. Donath, G. B. Sukhorukov, F. Caruso, S. A. Davis, and H. Möhwald, "Novel hollow polymer shells by colloid-templated assembly of polyelectrolytes," Angewandte Chemie International Edition, vol. 37, no. 16, pp. 2202-2205, 1998.

[11] I. L. Radtchenko, G. B. Sukhorukov, S. Leporatti, G. B. Khomutov, E. Donath, and H. Möhwald, "Assembly of alternated 
multivalent ion/polyelectrolyte layers on colloidal particles. Stability of the multilayers and encapsulation of macromolecules into polyelectrolyte capsules," Journal of Colloid and Interface Science, vol. 230, no. 2, pp. 272-280, 2000.

[12] J. F. Quinn, J. C. C. Yeo, and F. Caruso, "Layer-by-layer assembly of nanoblended thin films: poly(allylamine hydrochloride) and a binary mixture of a synthetic and natural polyelectrolyte," Macromolecules, vol. 37, no. 17, pp. 6537-6543, 2004.

[13] J. H. Wosnick, J. H. Liao, and T. M. Swager, "Layer-bylayer poly(phenylene ethynylene) films on silica microspheres for enhanced sensory amplification," Macromolecules, vol. 38, no. 22, pp. 9287-9290, 2005.

[14] K. Katagiri, A. Matsuda, and F. Caruso, "Effect of UVirradiation on polyelectrolyte multilayered films and hollow capsules prepared by layer-by-layer assembly," Macromolecules, vol. 39, no. 23, pp. 8067-8074, 2006.

[15] J. Seo, J. L. Lutkenhaus, J. Kim, P. T. Hammond, and K. Char, "Development of surface morphology in multilayered films prepared by layer-by-layer deposition using poly(acrylic acid) and hydrophobically modified poly(ethylene oxide)," Macromolecules, vol. 40, no. 11, pp. 4028-4036, 2007.

[16] D. G. Shchukin, I. L. Radtchenko, and G. B. Sukhorukov, "Synthesis of nanosized magnetic ferrite particles inside hollow polyelectrolyte capsules," Journal of Physical Chemistry B, vol. 107, no. 1, pp. 86-90, 2003.

[17] A. Voigt, H. Lichtenfeld, G. B. Sukhorukov, et al., "Membrane filtration for microencapsulation and microcapsules fabrication by layer-by-layer polyelectrolyte adsorption," Industrial \& Engineering Chemistry Research, vol. 38, no. 10, pp. 40374043, 1999.

[18] Y. Wu, T. Seo, S. Maeda, T. Sasaki, S. Irie, and K. Sakurai, "Circular dichroism induced by the helical conformations of acylated chitosan derivatives bearing cinnamate chromophores," Journal of Polymer Science B, vol. 43, no. 11, pp. 1354-1364, 2005.

[19] Y. Wu, T. Seo, T. Sasaki, S. Irie, and K. Sakurai, "Layered structures of hydrophobically modified chitosan derivatives," Carbohydrate Polymers, vol. 63, no. 4, pp. 493-499, 2006. 

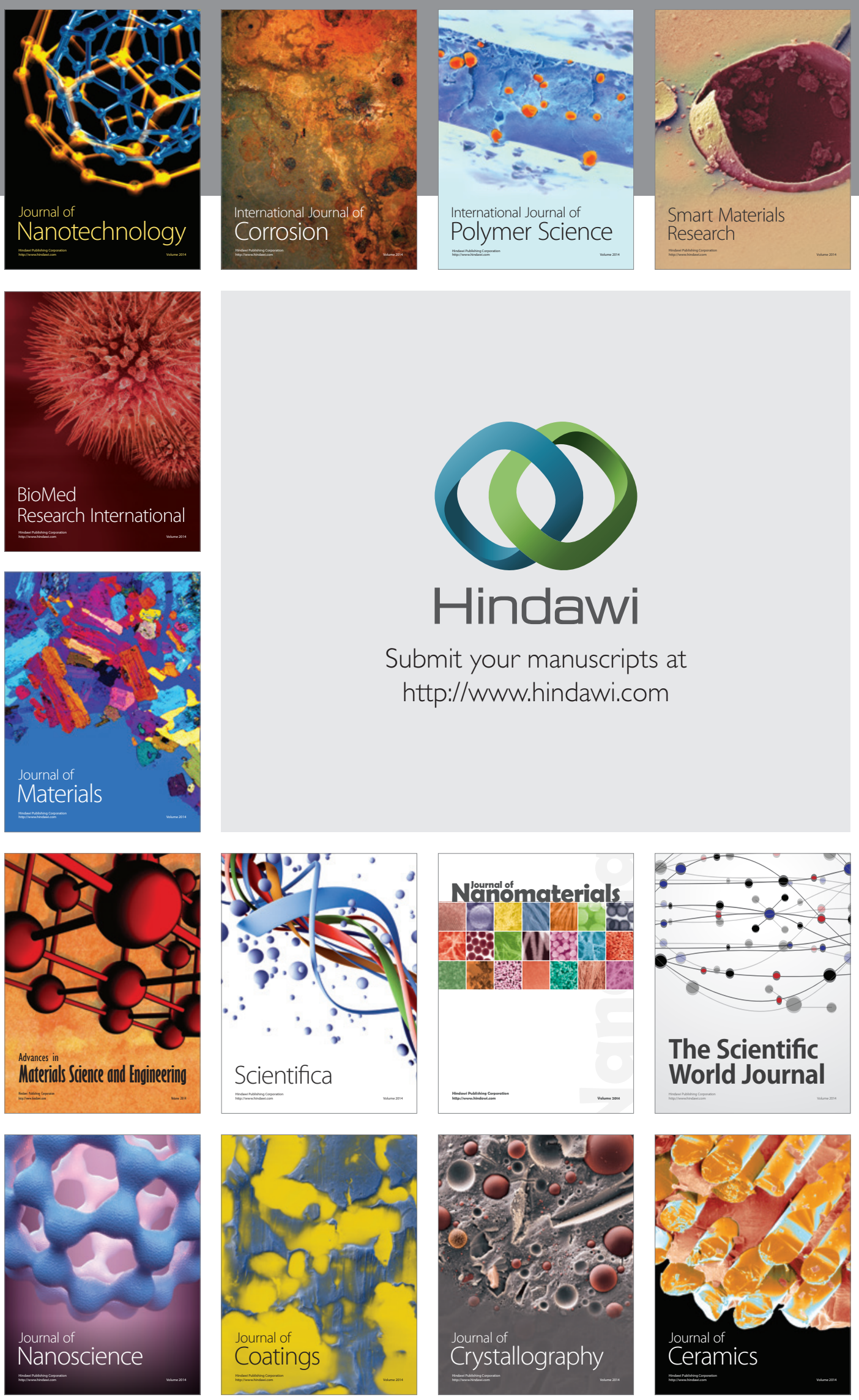

The Scientific World Journal

Submit your manuscripts at

http://www.hindawi.com

\section{World Journal}

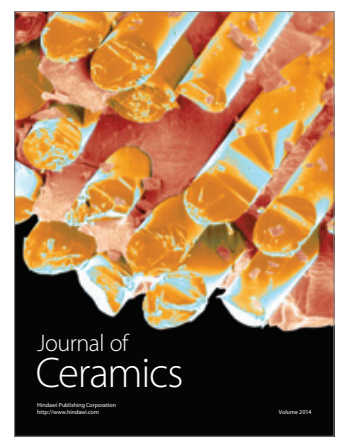

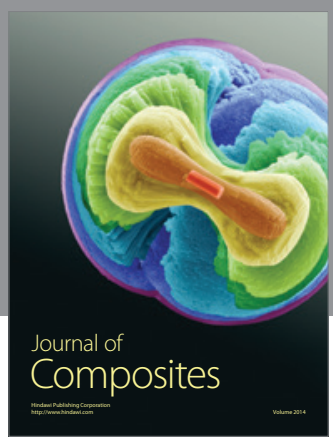
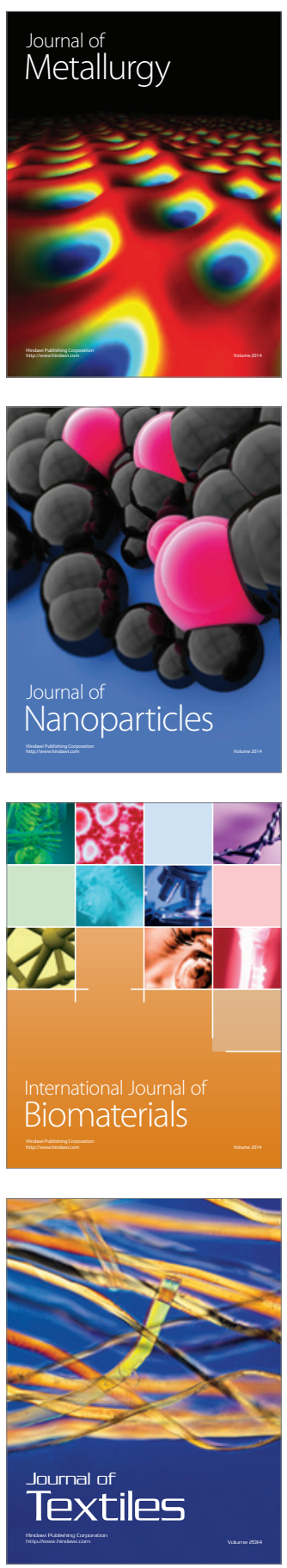